\title{
Multiscale behaviour of volatility autocorrelations in a financial market
}

\author{
Michele Pasquini and Maurizio Serva \\ Istituto Nazionale Fisica della Materia \& Dipartimento di Matematica \\ Università dell'Aquila, I-67010 Coppito, L'Aquila, Italy
}

\begin{abstract}
We perform a scaling analysis on NYSE daily returns. We show that volatility correlations are power-laws on a time range from one day to one year and, more important, that they exhibit a multiscale behaviour.
\end{abstract}

It is well known that stock market returns are uncorrelated on lags larger than a single day, in agreement with the hypothesis of efficient market. On the contrary, absolute returns have memory for longer times; this phenomenon is known in financial literature as clustering of volatility. In ARCH-GARCH models [1] [- volatility memory is longer than a single time step but it decays exponentially while empirical evidence is for hyperbolic correlations [4 9]. In this paper, we perform a scaling analysis of the standard deviation of a new class of observables, the generalized cumulative absolute returns. This analysis clearly shows that volatility correlations are powerlaws on a time range from one day to one year and, more important, that the exponent is not unique. This kind of multiscale behaviour is known to be relevant in the theory of dynamical systems, of fully developed turbulence and in the statistical mechanics of disordered systems (see [10] for a review) while it is a new concept for financial modeling.

We consider the daily New York Stock Exchange (NYSE) index, from January 1966 to June 1998, for a total of $N=8180$ working days. The quantity we consider is the (de-meaned) daily return, defined as

$$
r_{t}=\log \frac{S_{t+1}}{S_{t}}-\left\langle\log \frac{S_{t+1}}{S_{t}}\right\rangle
$$

where $S_{t}$ is the index value at time $t$ ranging from 1 to $N$, and $\langle\cdot\rangle$ is the average over the whole sequence. The underlying daily volatility $\sigma_{t}$ is not directly observable, but it is indirectly defined by $r_{t}=\sigma_{t} \eta_{t}$. It is assumed that the $\eta_{t}$ are identically distributed random variables with vanishing average and unitary variance. The usual choice for the distribution of the $\eta_{t}$ is the normal Gaussian. The observables directly related to the volatilities are the absolute returns $\left|r_{t}\right|$.

As pointed out by several authors [11 $[13]$, the distribution of returns is leptokurtic. In [12, it was firstly proposed a symmetric Lévy stable distribution and more recently in [13] it has been provided strong evidence for this fact. More precisely, in [13] it is shown that the distribution is Lévy stable for high frequency returns except for tails, which are approximately exponential. The estimation is that the shape of a Gaussian is recovered only on longer scales, typically one month.

Our analysis is on low frequency data, and first of all we want to verify that anomalous Lévy scaling is not effective in a range of time from one day to one year. We consider the cumulative returns $\phi_{t}(L)$, defined as the sum of $L$ successive returns $r_{t}, \ldots, r_{t+L-1}$, divided by $L$. Using NYSE data one can define $N / L$ not overlapping variables of this type and compute the standard deviation $\sigma(L)$. The standard deviation is independently computed for $L$ ranging from 1 to 250 (one year). Larger value of $L$ would imply insufficient statistics. Assuming that $r_{t}$ are uncorrelated (or short range correlated), it follows that $\sigma(L)$ has a power-law behaviour with exponent 0.5 for large $L$, i.e. $\sigma(L) \sim L^{-\beta}$ with $\beta=0.5$. The exponent for the NYSE index turns out to be about 0.49 (see fig. 1 and also see 14), according to the hypothesis of uncorrelated returns. This value of the exponent also ensures that Lévy scaling is not effective in this range of time.

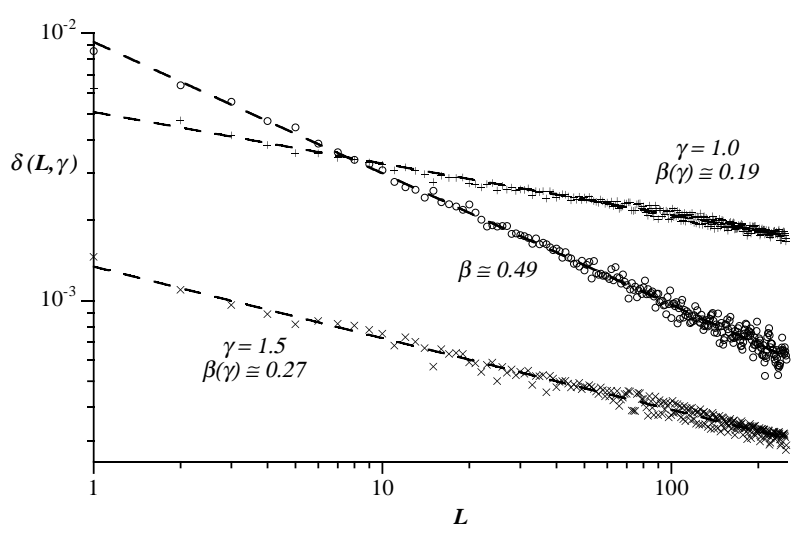

FIG. 1. Standard deviation $\delta(L, \gamma)$ of the generalized cumulative absolute returns (2) as a function of $L$ on $\log -\log$ scales for $\gamma=1$ (crosses) and $\gamma=1.5$ (slanting crosses), compared with the standard deviation of the cumulative returns (circles). The exponents of the best fit straight lines (dashed lines) are, respectively, $\beta(1) \simeq 0.19, \beta(1.5) \simeq 0.27$ and $\beta \simeq 0.49$.

Let us introduce the generalized cumulative absolute returns defined as

$$
\chi_{t}(L, \gamma)=\frac{1}{L} \sum_{i=0}^{L-1}\left|r_{t+i}\right|^{\gamma}
$$

where $\gamma$ is a real exponent and again, these quantities are not overlapping. If the $\left|r_{t}\right|^{\gamma}$ are uncorrelated, one should 
find that the standard deviation $\delta(L, \gamma)$ has a power-law behaviour with exponent 0.5 .

On the contrary, a power-law autocorrelation function with exponent $\alpha(\gamma) \leq 1\left\langle\left|r_{t}\right|^{\gamma}\left|r_{t+L}\right|^{\gamma}\right\rangle-\left\langle\left|r_{t}\right|^{\gamma}\right\rangle\left\langle\left|r_{t+L}\right|^{\gamma}\right\rangle \sim$ $L^{-\alpha(\gamma)}$, would imply that $\delta(L, \gamma)$ is a power-law with exponent $\beta(\gamma)=\alpha(\gamma) / 2$. For autocorrelations with exponent $\alpha(\gamma) \geq 1$ we would no detect anomalous scaling for the standard deviation $(\beta(\gamma)=0.5)$.

Our numerical analysis on the NYSE index shows very sharply that $\delta(L, \gamma)$ has an anomalous power-law behaviour in the range from one day to one year $(L=250)$. For example, for $\gamma=1$ we find $\beta(1) \simeq 0.19$, while for $\gamma=1.5, \beta(1.5) \simeq 0.27$ (see fig. 1). For larger $L$ the statistics becomes insufficient.

The crucial result is that $\beta(\gamma)$ is a not constant function of $\gamma$ in the range $-0.5<\gamma<+4$ (see fig. 2), showing the presence of different scales. The interpretation is that different $\gamma$ select different typical fluctuation sizes, any of them being power-law correlated with a different exponent.

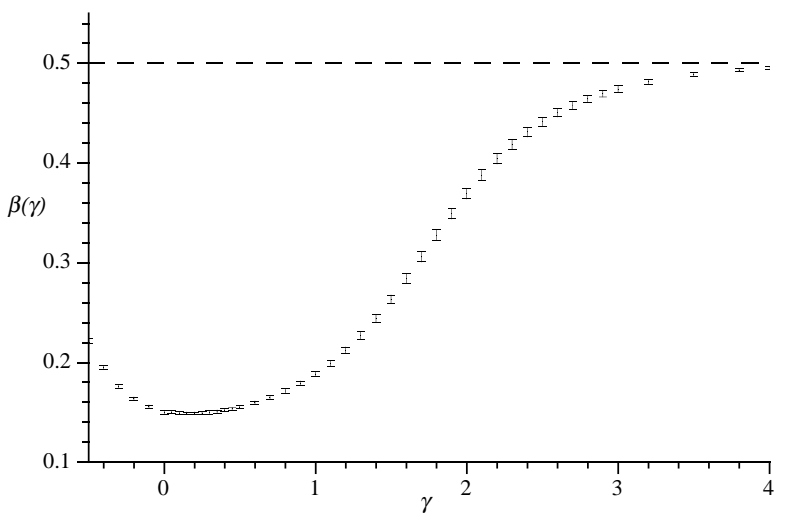

FIG. 2. Scaling exponent $\beta(\gamma)$ of the standard deviation $\delta(L, \gamma)$, where the bars represent the errors over the best fits. An anomalous scaling $(\beta<0.5)$ is shown in the range $-0.5<\gamma<+4$.

The longest correlation is for $\gamma=0.15(\beta(0.15) \simeq$ $0.15)$. The case $\gamma=0$ corresponds to cumulative logarithm of absolute returns.

In the region $\gamma \gtrsim 4$ the averages are dominated by only few events, corresponding to very large returns, and, therefore, the statistics becomes insufficient.

The anomalous power-law scaling can be directly tested against the plot of autocorrelations. For instance, the autocorrelations of $r_{t}$ and of $\left|r_{t}\right|$ are plotted in fig. 3 as a function of the correlation length $L$. Notice that the full line, which is in a good agreement with the data, is not a plot but it is a power-law whose exponent $2 \beta(1) \simeq 0.38$ is obtained by the previous scaling analysis of the variance. The autocorrelations for the return, as expected, vanish except for the first step $(L=1)$.

It should be also noticed that a direct analysis of the autocorrelations would not have provided an analogous clear evidence for multiscale power-law behaviour, since the data show a wide spread compatible with different scaling hypothesis.

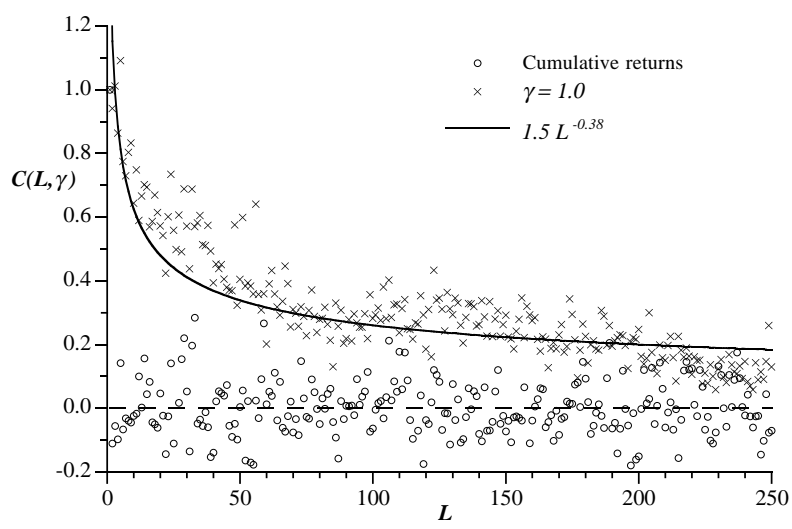

FIG. 3. Autocorrelation function of $\left|r_{t}\right|$ (crosses) as a function of the correlation length $L$, compared with the autocorrelation function of $r_{t}$ (circles). The data are in agreement with a power-law with exponent $2 \beta(1) \simeq 0.38$ in the first case, and absence of correlations in the second. In both cases the scale is fixed by autocorrelations equal to 1 at $L=1$.

In conclusion, we have found that scaling of variance of the generalized cumulative absolute returns implies multiscaling power-law correlations in financial indices. This result clearly suggests that models with exponential correlations, like ARCH-GARCH, are inadequate to describe the dynamics of financial markets, and they should be implemented to account for the coexistence of long memory with different scales.

[1] R.F. Engle, Econometrica 50 (1982) 987.

[2] P. Jorion, J. of Finance L (1995) 507.

[3] T.G. Andersen and T. Bollerslev, J. of Finance LIII (1998) 220.

[4] S. Taylor, Modelling financial time series (John Wiley \& Sons, New York, 1986).

[5] Z. Ding, C.W.J. Granger and R.F. Engle, J. of Empirical Finance 1 (1993) 83.

[6] R.T. Baillie and T. Bollerslev, J. of International Money and Finance 13 (1994) 565.

[7] P. De Lima and N. Crato, Economic Lett. 45 (1994) 281.

[8] R.T. Baillie, J. of Econometrics 73 (1996) 5.

[9] A. Pagan, J. of Empirical Finance 3 (1996) 15.

[10] G. Paladin and A. Vulpiani, Phys. Rep. 156 (1987) 147.

[11] P.K. Clark, Econometrica 41 (1973) 135.

[12] B.B. Mandelbrot, J. Business 38 (1963) 394.

[13] R. Mantegna and H.E. Stanley, Nature 376 (1995) 46.

[14] R. Mantegna and H.E. Stanley, Nature 383 (1996) 587. 